\title{
La negociación y el acuerdo: dos interpretaciones económicas de la justicia *
}

\author{
PAULETTE DIETERLEN \\ UNAM (México)
}

En un artículo aparecido en la revista Isonomía ${ }^{1}$ bajo el título de "Algunas consideraciones sobre la justicia distributiva» hacía una comparación entre ciertos elementos considerados característicos del mercado y aquellos que son típicos de las teorías de la justicia. Mencioné tres elementos: primero, la motivación que los defensores de cada uno de los conceptos -el mercado y la justicia- le atribuyen a la justicia; segundo, los principios que determinan la distribución de las cargas $\mathrm{y}$ los beneficios; $\mathrm{y}$, tercero, la condición necesaria para que se dé una distribución. Sin embargo, la distinción pertinente para abordar el problema de la justicia radica más bien en la manera como ésta se explica. Lo correcto sería separar las consideraciones económicas de la justicia de las consideraciones morales. En este trabajo me referiré especialmente a dos tipos de explicaciones que recurren a los modelos económicos para dar cuenta de lo que es una distribución justa: la negociación y el acuerdo ${ }^{2}$.

Primero, describiremos brevemente los tres elementos antes mencionados para después abordar, con más detenimiento, el tema de las transacciones.

El primer elemento que toman en cuenta los defensores de la visión económica de la justicia es la motivación «psicológica» que se adjudica a los agentes económicos: el interés propio. Si bien esta consideración puede rastrearse desde Hobbes, pasando quizá por Smith ${ }^{3}$, Bentham, Mill, hasta llegar a Friedman y Becker, fue Edgworth en 1881, en su obra Mathematical Psychics ${ }^{4}$, quien afirmó que el primer principio de la economía es que cada agente actúa motivado por su interés. La palabra «interés», sin duda, se refiere a un concepto que fue acunado por algunos pensadores de los siglos xvi y xvI, como Locke y Hume, y que más tarde fue desarrollado por Bentham y Stuart Mill: el concepto de utilidad. En la época victoriana, los filósofos y los economistas se refirieron a la utilidad como un indicador de bienestar global de las personas, como un indicador del placer que generaba la obtención de algo que era deseado. Pensaban que la utilidad era una medida numérica de la felicidad de una persona y que, con base en ella, se podía deducir que las personas hacian elecciones para maximizar su utilidad, esto es, para ser lo más felices posibles.

Debido a ciertos problemas conceptuales como es la asignación de una medida a la cantidad de utilidad asociada con elecciones distintas, los economistas se desvin. cularon del concepto de utilidad y, en su lugar, tomaron el de preferencia. Las preferencias pueden presentarse numéricamente asignándole un valor más alto a la alternativa preferida.

El segundo elemento de la visión económica de la justicia es el principio de utilidad que determina la distribución de los bienes y las oportunidades. Para el utilitarismo social clásico, una sociedad está

* Agradezco a Elisabetta di Castro y a Alejandro Tazzer la ayuda que me brindaron para hacer este trabajo. 
bien ordenada y es justa cuando sus instituciones están conformadas de tal manera, que alcanzan el mayor balance neto de las utilidades individuales sumadas, para obtener una economía llamada del kbienestar». Este cálculo social requería de comparaciones interpersonales $y$, tal como lo afirma Sen: «De hecho, el criterio tradicional de la economía de bienestar solía ser el utilitarista: valorar el éxito por el tamaño de la suma total de utilidad creada -al considerar que nada más tenía un valor intrínseco- ${ }^{5}$. Según este autor, la idea de la utilidad social se limitó aún más cuando, «en la década de 1930, Lionel Robbins (1935-1938) dirigió un ataque a las comparaciones interpersonales... Sea lo que fuere, con la aparición de la opinión anti-ética, al abandonar la economía del bienestar las comparaciones interpersonales de utilidad, el criterio sobreviviente fue el de la optimalidad de Paretom ${ }^{6}$.

Dos ideas contribuyeron a limitar el alcance explicativo de la economía del bienestar: primero, el teorema de imposibilidad de Arrow que, como veremos más ađelante, demostró que de la suma de preferencias individuales, las cuales son transitivas, no podemos pasar a establecer una preferencia social que sea también transitiva; segundo, las funciones de utilidad social que intentan sumar juicios morales distintos ${ }^{7}$. En lugar de hablar de la suma y el promedio de utilidades, los teóricos de la justicia prefirieron hablar de la negociación. Ésta y el criterio de optimalidad de Pareto, como también veremos más adelante, abrieron las puertas para el estudio de las transacciones y distribuciones, piedra angular de la justicia distributiva económica. Otro problema que surgió con las relaciones interpersonales es que violentaban la libertad del mercado: generalmente, el bienestar general óptimo no coincide con el resultado que se obtiene mediante la competencia perfecta. Por ello, algunas personas pueden pensar que es irracional la redistribución con la que se pretende hacer coincidir el resultado de la interacción del mercado y las exigencias utilitaristas, puesto que es necesario recurrir a argumentos morales en la explicación de por qué, con el objeto de incrementar la utilidad social, podemos reducir la utilidad de algunas personas para asignárselas a otros ${ }^{8}$.

El tercer elemento que se encuentra en esta clase de explicaciones es una condición inseparable de toda distribución: la eficiencia. La eficiencia es una situación económica que se logra mediante un mercado competitivo. Por una parte, el mercado nos señala cuánto se produce comparando el número de personas que desean un bien con el número de personas a las que se les paga por proveer el bien. Por otra parte, en un mercado competitivo cada persona paga el mismo precio por un bien, y la tasa marginal de sustitución entre un bien y «todos los demás bienes» es igual al precio del bien.

El tema del mercado nos lleva a considerar dos propuestas sobre las transacciones justas: la negociación y el acuerdo. Los teóricos de la negociación y del acuerdo intentan explicar las condiciones de posibilidad que tienen dos personas de distribuir un bien escaso sin necesidad de recurrir a un árbitro. Entenderemos, con Brian Barry, la justicia como «la distribución de derechos y privilegios, de poderes y oportunidades y de la disposición de los recursos materiales» ${ }^{9}$. Las posiciones que examinaremos traducen los elementos citados en la definición de la justicia a una función de utilidad, de tal modo que los conceptos como derechos, deberes, privilegios, etc., pueden explicarse recurriendo a la utilidad que reportan a los individuos. Se trata de dos teorias que pertenecen a lo que el propio Barry llama justicia considerada como ventaja mutua.

Si bien los defensores del análisis económico de la justicia fracasaron al derivar la noción de «utilidad social» recurriendo al concepto de utilidad interpersonal, sí 
pudieron solucionar el problema de las transacciones recurriendo a los instrumentos teóricos de la teoría de juegos. Dicha teoría parte de una noción de racionalidad como la elección de los mejores medios para alcanzar ciertos fines, independientemente de cuáles sean éstos. Los individuos enfrentados a otros hombres deben considerar las estrategias que les reporten la mayor utilidad, así como pensar que los otros harán lo mismo. El objeto del juego es llegar a un punto de equilibrio o de acuerdo; son ejemplo de ello la teoría de la negociación de Nash y del acuerdo de Gauthier.

\section{La negociación}

Para explicar la visión de la justicia como negociación empezaremos por definir lo que es el óptimo o el estado de frontera de Pareto. Un estado social se describe como óptimo en el sentido de Pareto si y sólo si no se puede aumentar la utilidad de algún agente sin reducir la utilidad de otro. Para entender cómo se lleva a cabo una distribución según Pareto, recurriremos a la explicación que nos proporciona Brian Barry 10. Una distribución se considera justa si el resultado de una transacción cntrc dos agentes representa una ganancia en comparación con lo que cada uno pudiera haber obtenido si persistiera una situación de conflicto. El proceso para determinar un resultado justo debe dividirse en dos partes. La primera consiste en establecer «el punto de desacuerdo» que es el resultado al que llegarian los agentes si no sc lograra un acuerdo. La segunda se refiere al movimiento que deben hacer los agentes para llegar wa un puntom en el que no sólo se preserve la ventaja que tenían en cl estado de desacuerdo, sino también que esté en el grupo de resultados «eficientes», lo que significa que uno de ellos no puede estar mejor sin que el otro empeore. En otras palabras, los agentes se mueven de un estado de desacuerdo a uno eficiente. Esta situación suele llamarse «la frontera de Pareto». Varios autores ${ }^{11}$ han coincidido en que el problema con el principio de eficiencia de Pareto es que en una negociación existe una gama amplia de posibilidades de distribución que cumplen con las exigencias del «punto de frontera de Pareto» y que las diversas distribuciones son ajenas a la situación de los agentes que llevan a cabo la negociación. Una distribución en la cual uno de los involucrados en la negociación tenga $10.00 \$$ y el otro $90.00 \$$ puede ser tan eficiente como otra en la que los dos terigan $50.00 \$$. Ahora bien, Barry nos explica ${ }^{12}$ cómo Nash intentó resolver el problema de la desigualdad recurriendo al criterio de utilidad que manejaron von Neumann y Morgenstern para explicar la elección racional que deben tomar los individuos en una situación de subasta hipotética ${ }^{13}$. Según ellos, es posible considerar un patrón de preferencias en un grupo de posibles distribuciones alternativas. Hay una forma de asignar las utilidades de ciertas distribuciones que nos permite describir la conducta de los individuos como la búsqueda de la maximización de la utilidad esperada ${ }^{14}$. La teoría que considera de esa forma las utilidades permitió explicarlas «en términos de la importancia relativa (utilidad relativa) que las personas le asignan a posibles pérdidas o posibles ganancias de dincro o de otros bienes no económicos» 15 .

Fl criterio de utilidad consiste en asignar una utilidad a los estados en los que se encuentran los individuos, así como a los estados en los que se encontrarán una vez hecha la ncgociacion. Este criterio también nos permite incluir la información que tiene un agente sobre las utilidades del otro. Por ejemplo, una persona rica puede scr indiferente entre obtener $50,00 \$$ seguros y una oportunidad igual de tener $100,00 \$ 00,00 \$$, en clonde la utilidad de tener $0,00 \$$ es de 0 , la de tener $100,00 \$$ 
es de $1, y$ la de tener $50,00 \$$ es de 0,5 . Una persona pobre puede ser indiferente entre tener $25,00 \$$ seguros y una oportunidad igual de tener $100,00 \$ 00,00 \$$, y la utilidad que le reporta tener $25,00 \$$ es también de 0,5 . La conclusión es que los dos tienen la misma utilidad, aunque el primero por adquirir $50,00 \$$ y el segundo tan sólo 25,00 \$. La conclusión de Nash es que, aun cuando el pobre necesite más el dinero, con la distribución puede igualar su utilidad con la del rico; por ello se trataría de una transacción justa. La diferencia básica entre «la frontera de Pareto» $y$ «el equilibrio de Nash» es que en este último se necesita igualar la utilidad producida por la transacción.

Para comprender la solución de Nash es conveniente resaltar cuatro elementos: 1) existe un punto de conflicto a partir del cual se calculan las ganancias que se obtendrán con la negociación; 2) la solución debe darse dentro de sla frontera de Paretow; 3) es necesario que exista una solución en la que se estipulen las ganancias que se obtienen si pasamos del epunto de conflicto» al de la «frontera de Pareto»; 4) la información que los agentes requieren para hacer la negociación se reduce a las utilidades que reporta la propia negociación a cada uno de los agentes.

Según Nash, es posíble llegar a una negociación si se dan tres condiciones. La primera es que la unidad con la que se mide la solución debe ser la misma con la que se mide la utilidad. La solución de Nash aborda las unidades de utilidad multiplicando todas las utilidades: por ejemplo, si se incrementan las utilidades del hombre pobre por un factor de 10 , la solución tiene que darnos la misma división del dinero, ya que los productos de la utilidad de los dos agentes deben respetar la misma proporción ${ }^{16}$. La segunda es la simetría de las utilidades: si los esquemas de utilidades de los agentes son idénticos, entonces el resultado tiene que dar una utilidad igual ya que éstos se miden de la misma forma que los esquemas. La tercera condición es la independencia de las alternativas irrelevantes; esto significa que un individuo elige entre un grupo fijo de alternativas, el cual es independiente de la existencia de otras alternativas fuera de dicho grupo ${ }^{17}$. Si se cumplen las condiciones y las soluciones, «el principio de Nash» representa la mejor alternativa que Ios individuos pueden elegir. Retomemos el ejemplo que nos proporciona Barry ${ }^{18}$. Un excéntrico millonario deja una herencia de 1.000 .000 de dólares a dos individuos, $P$ y $R$; la condíción para que la reciban es que puedan negociar su distribución; si en tres meses no llegan a un acuerdo, el dinero pasará a manos del Estado. La situación de $\mathrm{P}$ y de $\mathrm{R}$ es muy distinta ya que el primero tiene una deuda de 100.000 dólares con la mafia, mientras que $R$ ya es lo suficientemente rico como para que la obtención de 900.000 dólares le permita llevar una vida similar a la de Onassis. Tambiên conoce la situación de $\mathrm{P}$ y no tiene escrúpulos en tomar ventaja de ella. $\mathrm{Ni}$ a $\mathrm{P}$ ni a $\mathrm{R}$ les conviene que no haya negociación aunque $R$ no va a aceptar una división de 500.000/500.000, como tampoco $\mathrm{P}$ va a aceptar una división de $900.000 / 100.000$. El equilibrio se logrará dependiendo de la utilidad que representa para $P$ pagarle la deuda a la mafia y a $R$ el vivir como Onassis. El punto de negociación es «un equilibrio de Nash». La ventaja en esta situación es que, como vimos anteriormente, cada individuo asigna una utilidad relativa a las pérdidas o ganancias que oblendrá con la negociación. Si P otorga una gran utilidad a la necesidad de pagarle a la mafia, su situación en el intercambio será mucho más vulnerable. Por otro lado, si $\mathrm{R}$ afirma su desinterés por vivir una vida como Onassis, su situación en la negociación será más fuerte.

Aquí es pertinente mencionar que muchos economistas interpretan las convenciones sociales como la repetición de un jucgo producido por «el equilibrio de 
Nash». Por ejemplo, si en ciertas calles en el centro de Nueva York, en horas punta, todos los hombres de negocios caminasen por el lado derecho de la acera, lograrían un punto de equilibrio de Nash, ya que le asignan una gran utilidad a llegar temprano al trabajo. Esto no ocurriría si caminaran en sentido contrario a los demás ${ }^{19}$.

Ahora bien, después de analizar el mecanismo de distribución de Nash, es importante considerar la justicia que existe en la negociación. Para ello es necesario recurrir a dos argumentos. El primero consiste en afirmar que los dos negociadores tienen ciertos derechos que pueden ejercer de la forma que más les convenga. Tienen la posibilidad de emplear esos derechos o de abandonarlos a cambio de algo que deseen con más fuerza. El segundo se refiere a que el resultado de la negociación debe ser igualmente justo al que se daría si existiera un árbitro que, tomando en cuenta las utilidades esperadas, llevara a cabo la distribución ${ }^{20}$.

Sin embargo, uno de los problemas que surgen con las soluciones de Nash es que se necesita establecer un límite a los intercambios puesto que existen ciertos bienes, como ciertos derechos fundamentales, que no pueden ser intercambiables. Para decidir cuál es el límite entre lo negociable y lo que no lo es, necesitariamos a su vez de un procedimiento de negociación que no cumpla con las estipulaciones de Nash. Quizá se necesita de acuerdos morales previos a la propia situación de negociación, tal como lo establece la teoría de Gauthier que examinaremos a continuación.

\section{El acuerdo}

Gauthier, al igual que Hobbes, parte de las ventajas de la vida cooperativa para explicar su posición de que la moralidad no es más que un acuerdo que hacen los hombres para obtener ventajas. Es célebre el párrafo donde Hobbes expresó esta idea: «(sin la cooperación) no hay lugar para la industria; porque el futuro de la industria es inseguro. $Y$, por consiguiente, tampoco cultivo de la tierra; ni navegación, ni uso de los bienes que pueden ser impor* tados por mar; ni construcción confortable; ni instrumentos para mover y remover los objetos que necesitan mucha fuerza; ni conocimiento de la faz de la tierra; ni cómputo del tiempo; ni artes; ni letras; ni sociedad; sino to que es peor que todo, miedo continuo, y peligro de muerte violenta; y para el hombre una vida solitaria, pobre, desagradable, brutal y corta ${ }^{21}$. Sin embargo, Gauthier se separa de Hobbes al considerar que los individuos no necesitan de una autoridad para lograr acuerdos y para establecer mecanismos que los lleven a cumplirlos. Para el primero, el mercado perfectamente competitivo es el mecanismo que se encarga do regular tanto los acuerdos iniciales como su cumplimiento, ya que es «una lente a través de la cual la moralidad puede apreciarse más claramente. Si el mundo fuera un mercado semejante, la moral no sería necesaria... El mercado y la moral comparten la conciliación no coercitiva del interés individual y el beneficio mutuo" 22 .

Gauthier distingue entre una negociación y un acuerdo o una conducta cooperativa. En la negociación, la conducta de cada persona se caracteriza por responder, «maximizando» utilidades, según las cxpectativas que un agente se forma de la conducta de los demás; en el acuerdo, la conducta de cada persona se refiere a una respuesta «optimizadora» de acuerdo con las expectativas que se forman de la conducta de los demás; dicha conducta se basa en una estrategia conjunta que es elegida por todos. La diferencia entre la conducta maximizadora y la optimizadora consiste en que, mientras que la primera se refiere a la mejor alternativa que cada uno de los agentes puede lograr, la segunda se caracteriza por ser la mejor alternativa que un individuo obtiene después de haber 
hecho una concesión. Una concesión «es el ofrecimicnto que hace un futuro cooperador de aceptar una utilidad particular en vez de la que pretendias ${ }^{23}$. La cooperación surge cuando existen un conjunto de situaciones de negociación en las que los individuos calculan las ganancias que se dividirán después de que se haya llevado a cabo el acuerdo y la pérdida que implica hacer ciertas concesiones. Así, la racionalidad de la cooperación se refiere a las aportaciones que puede hacer un individuo a la mesa de la negociación una vez que se ha elegido una estrategia conjunta que permita desarrollar interacciones independientes ${ }^{24}$.

En la cooperación existen dos etapas: a) cada una de las partes presenta sus demandas y propone para la aceptación mutua un resultado o una estrategia conjunta. Como por lo general dichas demandas son incompatibles, es necesaria una segunda etapa, $b$ ) cada parte o alguna de las partes ofrece una concesión renunciando a alguna porción de su demanda original y propone un resultado alternativo. El proceso de concesión y demandas continúa hasta que se logra establecer un conjunto de propuestas mutuamente compatible, salvo que exista un desacuerdo insuperable 25 .

Ahora bien, uno de los obstáculos que es necesario superar surge de la propia racionalidad de la acción cooperativa. La teoria de juegos nos enseña que uno de los problemas de la acción cooperativa es que la estrategia dominante consiste en no cooperar: la racionalidad nos dicta que si nos abstenemos de cooperar obtenemos los beneficios de la cooperación de los otros sin pagar el costo de la cooperación ${ }^{26}$. Para que la conducta cooperativa sea racional es necesario encontrar un principio semejante al de la maximización de utilidad que caracteriza a la elección individual, asî como también descubrir un principio que nos permita encontrar la racionalidad del resultado obtenido por el ejercicio de un conjunto de estrategias y de las acciones que nos lleven a respetar los acuerdos. Una forma de hacerlo consistiría en derivar un orden de preferencias sociales del orden de preferencias individuales de quienes participan en la interacción. Sin embargo, nos dice Gauthier, Arrow demostró, mediante el teorema de imposibilidad, que esto no es posible. El argumento es el siguiente ${ }^{27}$ :

1. Si cada individuo prefiere el resultado $X$ al resultado $Y$, la sociedad prefiere $X$ a $Y$.

2. No hay ningún individuo cuyas preferencias sean automáticamente las preferencias de la sociedad, sean cuales fueren las preferencias de los demás.

3. Las preferencias sociales sobre cualquier resultado dependen sólo de las preferencias individuales sobre el resultado.

Para Gauthier, la aportación de Arrow consistió en descubrir que, puesto que necesitamos que la elección cooperativa seleccione un resultado óptimo, no podemos rechazar la condición 1 . Puesto que necesitamos que la elección cooperativa refleje las preferencias de cada individuo, no podemos rechazar la condición 2 . Y puesto que en esta proposición la preferencia social se basa en las preferencias individuales, y sólo en ellas, no podemos rechazar la condición 3 . De ahí que no podamos hacer derivar un orden de preferencias social de todas las configuraciones de los órdenes de preferencias individuales y que la sugerencia de pasar así de to individual a lo social no puede ser un buen arranque ${ }^{28}$.

Según Gauthier, el contexto de la elección cooperativa es completamente diferente por dos razones. Primero, porque la cooperación exige que los agentes actúen de una forma dinámica, es decir, que vayan cambiando sus demandas y sus concesiones dependiendo de las demandas y concesiones que hagan los demás. Por ello nos dice: 
«Suponer que los individuos han de considerar indiferentemente los resultados óptimos, de modo tal que la selección entre ellos sea sencillamente fortuita, es pensar que el proceso del acuerdo carece del carácter dinámico que realmente posees ${ }^{29}$. Segundo, porque la cooperación nos da un excedente cooperativo que va a ser compartido por todos. El excedente cooperativo es un conjunto de diferencias de utilidad, una correspondiente a cada cooperador, ninguna con valor negativo y todas iguales a la diferencia que existe entre la utilidad que proporciona la cooperación y la utilidad que garantiza la posición negociadora ${ }^{30}$. Por su parte, una demanda se define como «aquello que exige un futuro cooperador de un excedente cooperativo en el momento de iniciar la negociación" 31. Puesto que cada uno desea beneficiarse con el, nadie tiene interés en provocar el fracaso del proceso negociador, ya que esto llevaría al fracaso de la cooperación y por lo tanto a la desaparición dèl excedente. Así se eliminan los problemas de la estrategia dominante no cooperativa, ya que el que no coopera simplemente pierde las ganancias del excedente. Cada persona, al tratar de maximizar su propia utilidad y estar consciente de que los demás tratarán de maximizar la suya, llegará a aceptar una estrategia para lograr el excedente cooperativo. Aquel que haga la concesión obtendrá una utilidad de 1 , mientras que el que no lo haga se conformará con 0 . De esta manera, se tiene una medida de concesión relativa que, sin introducir ninguna comparación de utilidad interpersonal, nos permite comparar las concesiones de las personas que participan en una situación de negociación ${ }^{32}$. Cada resultado será admisible no desde el punto de vista de las utilidades que le brinda a cada persona, sino desde cl punto de vista de las concesiones que cada persona debe hacer si tuviera que determinar la estrategia conjunta que regularía la interacción cooperativa.
Gauthier aplica «el principio de Zeuthen» ${ }^{33}$ para fijar una regla que decida qué resultado debe ser aceptado, Este principio establece que la persona cuyo promedio entre el costo de la concesión y el costo del desacuerdo insuperable sea menor, debe ceder ante el otro, es decir, una persona con un menor grado de concesión debe ceder ante los demás. Si extendemos los alcances de esta regla a la negociación cntre varias personas, el principio establecería que, dados varios resultados posibles de ser seleccionados, algunas personas o todas las personas deben hacer ciertas concesiones. Un resultado debe elegirse si la concesión relativa mayor o máxima que se exige es lo menor o mínima posible, es decir, que no sea mayor que la concesión relativa máxima cxigida por ninguno de los otros resultados. Gauthier llama a esta regla «principio de mínimo máximo»o «principio de concesión relativa minimáximas 34

La racionalidad fundamental del principio de concesión relativa minimáxima se refiere a una comparación interpersonal de la proporción de la ganancia potencial que cada persona debe conceder ${ }^{35}$.

Las condiciones de esta cooperación o acuerdo racional son las siguientes:

1. La pretensión racional. Cada persona debe pretender el excedente que le proporcione la máxima utilidad, salvo que ninguna persona pueda pretender un excedente cooperativo si no fuera un participante de la interacción necesaria para alcanzarlo.

2. El punto de concesión. Dadas las pretensiones que satisfagan la condición 1, cada persona debe suponer que existe un punto de concesión factible que toda persona racional esté dispuesta a tomar en consideración.

3. La disposición a conceder. Cada persona debe estar dispuesta a tomar en consideración una concesión en relación con un punto de concesión si la magnitud rela- 
tiva de tal concesión no es mayor que la de la concesión mayor que el individuo supone que una persona racional está dispuesta a tomar en consideración (en relación con un punto de concesión factible).

4. Los limites de la concesión. Ninguna persona está dispuesta a tomar en consideración una concesión que esté en relación con un punto de concesión si no se lo exigen las condiciones 2 y $3^{36}$.

De esta manera Gauthier formula el principio de concesión relativa minimáxima: en cualquier interacción cooperativa, la estrategia conjunta racional queda determinada por una negociación llevada a cabo entre los cooperadores, negociación en la cual cada individuo presenta su pretensión máxima y luego ofrece una concesión cuya magnitud relativa no pucde ser mayor que la concesión minimáxima.

El principio de concesión relativa minimáxima desempeña una funcion triple en la argumentación. Primero, expresa el principio de maximización de la utilidad esperada dentro de la negociación; scgundo, determina el contenido formal de una negociación racional que es la estrategia conjunta; tercero, es el principio de la conducta racional en la interacción cooperativa, la interacción basada en la estrategia conjunta aceptada en la negociación. Cada individuo obra no para maximizar su propia utilidad, sino para alcanzar el resultado que es el objeto de la negociación y que le proporciona a cada persona una utilidad esperada no menor que la que podría esperar de su pretensión máxima y su concesión minimáxima ${ }^{37}$.

Ahora bien, según Gauthier, la interacción cooperativa forma parte de la justicia. Esto se debe a que, primero, al igual que el mercado, la cooperación racional excluye toda parcialidad; segundo, porque a diferencia del mercado, esa exclusión exige que cada cooperador restrinja su actividad maximizadora. La interacción cooperativa limita a las personas, les impide tomar ventajas de sus pares y las restringe imparcialmente, de un modo beneficioso para todos. Dada una posición inicial, la cooperación es justa si la estrategia conjunta en la que se basa es el resultado de una negociación justa entre los cooperadores ${ }^{38}$.

A diferencia de «la solución de Nash», aquí los individuos reflexionarían sobre aquellos aspectos de su vida que están dispuestos conceder y aquellos sin los cuales no pueden empezar la negociación. Tanto la cooperación como el acuerdo requieren que los individuos partan de un principio justo. Gauthier entiende por justicia: «la disposición que tiene el individuo a no sacar ventaja de sus semejantes, a no tratar de apoderarse de los bienes libres, de no imponer costos no compensadores siempre que el individuo suponga que los demás están igualmente dispuestos» ${ }^{39}$. Esta definición de la justicia nos permite garantizar los resultados del acuerdo.

Las preguntas que nos surgen al explicar la teoria de las transacciones justas de Gauthier son al menos las siguientes: primero, en relación a la garantía que tenemos de que todo acuerdo va a proporcionar un excedente; $y$, segundo, sobre si efectivamente la repartición de dicho excedente constituye una motivación suficiente para superar la tentación de las ganancias de una conducta no cooperativa tal como lo muestra la lógica de la acción colectiva.

\section{Conclusión}

Nos hemos referido a dos teorías de la justicia que se basan en el concepto de utilidad como elemento explicativo de la motivación de la conducta humana y que, en especial, parten del análisis de la conducta individual para explicar las transacciones. Según autores como Nash y Gauthier las instituciones que se encargan de distribuir los derechos, las prerrogativas, los deberes y las obligaciones únicamente 
tendrían que respetar las negociaciones y los acuerdos que llevan a cabo los individuos. Ahora bien, es necesario destacar que estas propuestas parten de una concepción del hombre magistralmente expuesta, en una de las Tanner Lectures on Human Values, por el premio Nobel en economía George J. Stigler:

Durante mucho tiempo - de hecho, durante muchísimo tiempo-, la teoría del interés propio (como la he interpretado cn las lineas de Smith) vencerá. En una serie de casos nada irrelevante, y tal vez aleatoria con respecto a las características sociales de los actores, la hipótesis del interés propio fallará; al menos con una interpretación sutil e impredecible del interés propio. Vaticino este resultado porque es el que con mayor frecuencia han encontrado los economistas, no sólo dentro de una amplia variedad de fenómenos económicos, sino también en sus investigaciones sobre el comportamiento matrimonial, en relación con el número de hijos, el comportamiento criminal, el religioso y otros comportamientos sociales. Creemos que el hombre es un animal maximizador de utilidad - aparentemente también lo son las palomas y las ratas-y hasta el presente no hemos encontrado información para descubrir una parte de su vida en la que invoque unos objetivos de comportamiento diferentes. De hecho, la prueba que he propuesto tiene un alcance muy limitado, porque la mayoria de los valores éticos no entran en conflicto con el comportamicnto individual maximizadior de utilidad ${ }^{40}$.

Sin embargo, más allá del valor que tienen los enfoques económicos de la justicia, hay que considerar la obra de muchos autores que han abordado el problema de la justicia desde un punto de vista moral. Ejemplo de ello lo constituye la obra de John Rawls. Tanto en Teoria de la justicia ${ }^{41}$ como en Liberalismo político ${ }^{42}$ ha intentado proponer otra solución al problema de la justicia. A diferencia de la propuesta de Nash -que exige el conocimiento de las utilidades que el otro adjudica a su elección-, Rawls priva a los individuos de toda información relevante para, de este modo, garantizar la imparcialidad. Frente al concepto de cooperación de Gauthier, este autor la concibe como una empresa en la que las personas racionales y razonables persiguen, junto con los demás, la consecución de su idea del bien. Finalmente, ante las teorías que explican la justicia con criterios consecuencialistas, rccurre a principios deontológicos que exigen un respeto irrestricto por los derechos liberales.

Quizá las teorías de la justicia que incorporan la moralidad como uno de los criterios para justificar ciertas distribuciones no tengan los recursos teóricos tan sofisticados como para poder cuantificar las preferencias, determinar curvas de utilidad, $y$, en gencral, aprovechar el desarrollo matemático como presupone la teoría de juegos, pero ciertamente se adecuan mejor a las intuiciones que tenemos de la justicia.

\section{NOTAS}

' Isonomia, Revista de Teorta y Filosofía del Derecho, núm. 2, México, D. F, abril 1995, pp. 69-85.

${ }^{2}$ En este trabajo abordaremos los enfoques que parten de las transacciones entre los individuns. Estas posiciones no incluyen acuerdos «institucionalesm.

3 Es interesante conocer la indicación de Sen sobre ciertas interpretaciones de Smith que reducen la motivación de los agentes a un interés egoísta. Sen piensa que la motivaciớn humana en Smith es mucho más compleja. Cfr. Amartya Sen, Sobre ética y economí, México, D. F, Alianza Editorial, Consejo Nacional para la Cultura y las Artes, 1992, pp. 39-45.

* Citado por $A$. Sen, aRational Fools*, en Philosoply and Economic Theon, Oxford, Oxford University Press, 1979, p. 87 .

5 A. Sen, Sobre ética y economia, pp. 48-49.

"Ibid.

7 Hal Varian, Intermediate Microeconomics. A 
Modem Approach, New York, W. W. Norton \& Company, $19 \%$, p. 532 .

-Cr. D. Gauthier, La moral por acuerdo, Barcelona, Gedisa, 1994, p. 176.

"B. Barry, Theories of Justice, vol. I, Berkeley, University of Calitornia Press, 1989, p. 292.

${ }^{19}$ Op. cit., p. 10.

"Cfr. B. Barry, op cit, p. 13. A. Sen, op. cit. p. 49. J. Rawls, Teoria de la justicia, México, Fondo de Cultura Económica, 1995, p. 74.

12 Tbid. Barry se basa en el articulo de Nash 4 The Bargaining Problents, Econometrica, 18, 1950 , pp. 155-162.

13 La solución de Nash evidentemente no es la única que se ha dado al problema. Barry discute otras propuestas como la de Harsanyi y la de Braithwaite. Cfr. op. cit., pp. 17-60. En este texto utilizamos el principio de Nash como uno de los ejemplos de una distribución en la que los agentes adquieren ventajas mutuas. La obra de Von Neumann y Morgenstem donde se encuentra la explicación de la maximización de utilidad esperada es The Theory of Games and Economic Behavior, Princeton, Princeton University Press, 1944.

is Cfr. K. Arrow, Social Choice and individual values, New Haven, Yale University Press, 1963, pp. 9-10.

is Harsanyi, Morality and the theory of rational behaviourn, en A. Sen y B. Williams, Utilitarianism and Beyond, Cambridge, Cambridge University Press, 1983 , p. 53.

16 B. Barry, op. cit. p. 17.

17 K. Arrow, op. cit. p. 27.
Ibiat, p. 23.

19 Andreu Mas-Colell, Michael D. Whiston y Jerry R. Grenn, Microeconomic Theony, Oxford, Oxford University Press, 1995, p. 249

al Cr. B. Barry, op. cit., p. 51.

2. Leviatán, Madrid, Editora Nacional, 1979, p. 225.

22 D. Gauthier, op. cit, p. 30.

23 D. Gauthier, op. cut, p. 192

24 Ibid., p. 182.

2.s Ibid.

76. Cir. I. Elster, Sour Grapes, Cambridge, Cambridge University Press, 1985, pp. 26-33.

27 lbid, p. 170.

2* Ibid, p. 171 .

29. Ibid.

ofr. op. cit, p. 192.

"Ibid.

32 Ibid., p. 186.

"El desarrollo del principio de Zeuthen se encuentra en su obra Problems of Monopoly and Economic Warfare, London, G. Routledge and Sons, 1930.

is lhid. p. 187 .

${ }^{35}$ lbid, p. 189.

35 Ibid, pp. 192-194.

37 Ibid, p. 196.

Is Ibid., p. 203.

${ }^{39}$ Ibid. p. 150

4) G. J. Stigler, El economista como predicador y otros ensayos, Barcelona, Edictones Folio, 1987, p. 46.

"México, Fondo de Cultura Económica, 1995.

42 Barcelona, Crítica, 1996. 